\title{
EDITORIAL
}

AFRICAN JOURNAL OF CLINICAL AND EXPERIMENTAL MICROBIOLOGY AJCEM/1833

COPYRIGHT 2018 https://dx.doi.org/10.4314/ajcem.v19i4.1

AFR. J. CLN. EXPER. MICROBIOL. 19 (3): 238-250
ISBN 1595-689X SEPTEMBER 2018 VOL19 No.4

Shttp://www.ajol.info/journals/ajcem

\section{INTERNAL AND EXTERNAL QUALITY CONTROL IN THE MEDICAL MICROBIOLOGY LABORATORY}

\author{
Fowotade $^{1,4}$, A., Fayemiwo ${ }^{1,3}$, S.A. , Bongomin ${ }^{2,3}$ F., Fasuyi ${ }^{4}$ T.O., Aigbovo ${ }^{1}$, O.A,. Adegboro ${ }^{5}$, B. \\ ${ }^{1}$ Department of Medical Microbiology and Parasitology, College of Medicine, University of Ibadan, Ibadan, Oyo state, Nigeria; \\ ${ }^{2}$ Department of Medicine, College of Health Sciences, Makerere University, Kampala, Uganda; ${ }^{3}$ Division of Infection, \\ Immunity and Respiratory Medicine, The University of Manchester, Manchester, M13 9PL, UK; ${ }^{4}$ Department of Medical \\ Microbiology and Parasitology, University College Hospital, Ibadan, Oyo state, Nigeria; ${ }^{5}$ Department of Medical \\ Microbiology, LAUTECH Teaching Hospital, Ogbomosho, Oyo State, Nigeria.
}

\begin{abstract}
Culture media play a very important role in bacteriology as they are used in the isolation, identification and antimicrobial susceptibility testing. It is essential that the quality of media be safeguarded to have a successful microbiology laboratory. Microorganisms usually show typical morphological appearance and properties on solid media. Variations in the composition of the medium may alter this appearance and properties. There is therefore a need to ensure good quality media, which is capable of giving satisfactory results by ensuring a proper quality management system. Often times, majority of laboratories prepare their media for routine diagnostics and research purposes. Therefore, it is essential that certain parameters of media are checked thoroughly before they are considered suitable for laboratory use. Control methods are discussed in details in this report.
\end{abstract}

CONTRÔLE DE QUALITÉ INTERNE ET EXTERNE DANS LE LABORATOIRE DE MICROBIOLOGIE MÉDICALE

Fowotade $^{1,4}$, A., Fayemiwo ${ }^{1,3}$, S.A. , Bongomin ${ }^{2,3}$ F., Fasuyi ${ }^{4}$ T.O., Aigbovo ${ }^{1}$, O.A,. Adegboro ${ }^{5}$, B.

1 Département de microbiologie médicale et de parasitologie, Collège de médecine, Université d'Ibadan, Ibadan, État d'Oyo (Nigéria); 2Depart of Medicine, Collège des sciences de la santé, Université Makerere, Kampala, Ouganda; 3Division de l'infection, de l'immunité et de la médecine respiratoire, Université de Manchester, Manchester, M13 9PL, Royaume-Uni; 4Département de microbiologie médicale et de parasitologie, University College Hospital, Ibadan, État d'Oyo (Nigéria); 5Département de microbiologie médicale, hôpital universitaire LAUTECH, Ogbomosho, État d'Oyo, Nigéria.

\begin{abstract}
ABSTRAIT
Les milieux de culture jouent un rôle très important en bactériologie, car ils sont utilisés dans les tests d'isolement, d'identification et de sensibilité aux antimicrobiens. Il est essentiel que la qualité des médias soit garantie pour avoir un laboratoire de microbiologie performant. Les micro-organismes présentent généralement une apparence morphologique et des propriétés typiques sur des milieux solides. Des variations dans la composition du support peuvent altérer cet aspect et ces propriétés. Il est donc nécessaire de garantir des supports de qualité, capables de donner des résultats satisfaisants en assurant un système de gestion de la qualité approprié. Souvent, la majorité des laboratoires préparent leurs médias à des fins de diagnostic et de recherche de routine. Par conséquent, il est essentiel que certains paramètres des supports soient soigneusement vérifiés avant de pouvoir être considérés comme pouvant être utilisés en laboratoire. Les méthodes de contrôle sont discutées en détail dans ce rapport.
\end{abstract}

\section{BACTERIOLOGY LABORATORY}

\section{Source of Media}

In the past, bacteriologic media were prepared from the basic chemical ingredients but this is no longer a common practice as many laboratories no longer do this. Rather, commercially-prepared dehydrated media which requires only the addition of water in for its reconstitution and use are now routinely used by most laboratories. Hence, the responsibility of quality control is that of the manufacturer however the quality of the media must be tested after its preparation due to changes which can occur following reconstitution and sterilization(1). For these dehydrated media, quantities ordered should be used up within 6 months or at most one year. It should be stored in a cool, dark, well ventilated place with the caps of all containers sealed tightly as dehydrated media are hygroscopic. Date of receipt and opening of media should be recorded. Darkened or caked dehydrated media must be discarded (2). 
Often times, certain additives such as blood, serum, growth factors, specific vitamins, antibiotics are used in the preparation of media especially for fastidious organisms. The quality of blood used in the preparation of blood-containing media is essential to the performance of the media. The sterility, viscosity, homogeneity and colour of the blood should be carefully checked prior to its use for media preparation (3). In the case of other additives, the certificate of analysis and sterility conditions should be considered prior to usage while heat labile additives whose sterility is uncertain can be sterilized by membrane filtration (4). Commercially-prepared, ready to use media are also available and utilized by some laboratories. The quality control maintenance of such media is the responsibility of the manufacturer. A written assurance must be supplied by the manufacturer stating that Clinical and Laboratory Standards Institute (CLSI) standards were followed and this verification must be maintained along with the QC protocol of the laboratory for as long as the laboratory uses the specified media.4 In addition, a certificate of analysis stating the expiration date, storage conditions should accompany all commercially-prepared, ready to use media along with quality control organisms used in growth supporting ability and selectivity testing (5). However, the laboratories also need to inspect each shipment of media for its properties $(1,4)$.

\section{Quality and Quantity of Water}

The quality of any media depends directly on the quality of raw materials used in its preparation with water being the most important raw material (6). Tap water should not be used in media preparation due to the presence of impurities rather distilled and/or deionized water should be used.1 The copper content, $\mathrm{pH}$ and conductivity of the water should be checked prior to its use in the preparation of culture media. Ideally, water used should have no copper in it because of its inhibitory effect on microorganisms. The $\mathrm{pH}$ of the water should be slightly acidic but not less than 5.5 while the conductivity should not be less than $15 \mu \mathrm{S}$ (microsiemens) (3). The amount of water added while reconstituting media should be carefully measured in order to have adequate gel strength $(1,3)$.

\section{Weighing}

Weighing balances used in the measurement of dry materials must be accurate as weighing errors significantly alter the composition of the final product (1).

\section{Quality of Glassware}

Glassware used in media preparation should be made of borosilicate glassware, as glassware made of soda can leach alkali into the media resulting in an alteration of the $\mathrm{pH}$ of the media.3 Glassware used should be carefully cleaned as residues on glass may be inhibitory in some fastidious organisms (1).

\section{Quality of Sterilization}

The process of sterilization also plays an important role in the quality of culture media. Generally, culture media is sterilized by autoclaving. But, certain parameters must be met in order to achieve adequate sterilization. These include the autoclaving time, temperature and pressure as well as close regulation of the quantity of media sterilized. Heat treatment of complex culture media may result in destruction of nutrients present in the media either by direct thermal destruction or reaction between its components. Sterilization of media at too high a temperature or for too long or both may result in the deterioration of some constituents of the media thereby rendering it unsuitable for use (1). Hence, it is necessary to minimize the heating damages by optimizing the heating process. The autoclave time to achieve sterility at a temperature of $121^{\circ} \mathrm{C}\left(250^{\circ} \mathrm{F}\right)$ is 15 minutes while the autoclave pressure is 15 psi (100KPa).4 The volume of media in a sterilization batch should be small, ideally 2 Litres (3).

Regular checking of the sterilization process by the use of indicators is essential; the temperature and pressure should also be monitored constantly. Sterilization indicators are used to check the efficacy of the sterilization process. Biological sterilization indicators such as the spores of Bacillus stearothermophilus can be used to check the spore killing efficacy while the chemical indicator such as Bowie Dick test can be used to check the efficiency of the sterilization process (3).

The physical appearance of the media should be inspected after sterilization as there might be alteration in its appearance. The following may be observed: turbidity or a precipitate indicating that some constituent has come out of the solution; darker than normal colour indicating overcooking of sugar containing media, incorrect mixture of constituents or wrong $\mathrm{pH}$; lighter than normal colour indicating incorrect mixture of constituents or wrong $\mathrm{pH}(1)$.

\section{Dispensing}

The quality of the Petri dish used in the preparation of the media is also important. Petri dishes are normally sterilized with ethylene oxide (Eto) or gamma irradiation. Eto sterilized Petri dishes should be checked for residual Eto toxicity which may inhibit the growth of microorganisms. The maximum 
permissible limit for residual Eto is $1 \mu \mathrm{g} / \mathrm{g}$. Residual Eto can be measured by standard gas chromatographic method (3). Aseptic technique is essential while dispensing media and accurate volume should be dispensed. About 25-30 ml of media should be dispensed into a $100 \mathrm{~mm}$ Petri dish. Inaccurate measurement of the amount of media may result in too shallow or too deep media rendering it unsuitable for use(1). Media should be dispensed into Petri dishes on a flat, level surface in order to have equal filling.

\section{Physical Parameters of the Media}

The gross physical appearance of media is an indicator of its quality. All media, either userprepared or commercially-prepared should be screened for physical parameters such as colour, consistency, depth, smoothness, excessive bubbles or pits, unequal filling of Petri dishes, cracked medium in plate, clarity, freezing( seen as a crystalline pattern on the surface of the medium), presence of leakage and visible contamination. All these are checked visually by the naked eye. However, depth of the medium and unequal filling of Petri dishes can be checked at four points. The four points are the two ends of the two diameters of the plate which are at right angles to each other. The depths at the four points are noted, the mean thickness is calculated and reported as the mean thickness of the medium in the plate which must be $4.0 \pm 0.2 \mathrm{~mm} .3$ Blood based media should be checked for signs of haemolysis. There should be no deviation from the normal colour of the media. It should be free of moisture but should have no signs of drying at the edges, Petri dishes should have not be broken or cracked.7 The $\mathrm{pH}$ of the medium must be checked while preparing the medium before and after autoclaving using a standard $\mathrm{pH}$ meter after calibration with standard buffers (3).

The gel strength which is an indication of the level of solidification of the agar within the medium is also measured. This is done with the aid of a tripod stand with a central rod that is used to impart pressure on the agar. The lower end of the rod has a spherical portion which rests on the surface of the medium while the upper end of the rod has a platform on which standard weights are placed. The spherical part of the central rod is placed on the medium and then weights are placed on the upper platform one after another and observed for a while. This process is continued until the agar breaks under the weight of the central rod. The gel strength can then calculated by deducting the weight of the central rod from the total weight. The force imparted by the rod on the agar surface is calculated by the formula: Wpr2 where $\mathrm{W}$ is the weight of on the platform, $\mathrm{r}$ is the radius of the spherical part of the lower end of the central rod and $\mathrm{p}$ is 3.14 (a constant). The acceptable gel strength is about 300-500 dynes/cm (2).

\section{Contamination}

This is a very important indicator of the media quality, it is also known as sterility testing. Each batch of media must be checked for contamination before it is declared fit for laboratory use. The common technique in sterility testing is randomly picking a small number of media (usually between 1$5 \%$ of the batch) at random and placing in an incubator at a selected temperature and time depending on the type of media though for general purpose $30-37^{\circ} \mathrm{C}$ for $48-72$ hours is typical. After which the plates are checked for microbial growth. If there is no growth the batch is declared fit for use, the samples used in the sterility testing should be discarded as they are unsuitable for inoculation due to the dehydration that occurs after up to 48 hours incubation. If there is growth, the process is repeated. If there is growth again, the batch is inferred to have been contaminated. It is recommended that a batch be discarded when there is more than $10 \%$ contamination (8). Alternatively, it has been suggested that contamination can be checked by leaving the whole batch of prepared media at room temperature for at least three days after which it is examined for visible growth (3).

\section{Growth Supporting Ability}

Growth supporting ability is the most important parameter when conducting quality control of a media. In order to demonstrate the growth promoting ability of media, a panel of microorganisms is required. Preferably, the recommended microorganisms should be traceable to a reputable culture collection such as the American Type Culture Collection. The media should be inoculated with overnight cultures of pure, well defined and appropriate test strains of the organism (9). When testing new lots of media, the results should be examined both qualitatively and quantitatively while using standard inoculating procedures. The previous batch and a new batch should be simultaneously inoculated. Results of previous batches of the same media should be compared with that of a new batch. During testing, some priorities must be established such as beginning the testing with media that are most likely to demonstrate deficiencies. Top priority should be accorded to blood, chocolate and Thayer Martin agars and secondary priority should be given to selective enteric media such as MacConkey, EMB, XLD and bile salt containing agars (1). Testing of selective media should include inoculation of organisms supported by the media and organisms inhibited by the media in order to demonstrate its 
selective nature. To demonstrate the inhibitory effect, the medium can be challenged with a heavy inoculum of the organism, since if the medium can inhibit the growth of a large inoculums it will also prevent the growth of the small number of organisms present in specimens.1 When testing media used to identify a biochemical response such as hydrogen sulphide production or fermentation, species or strains of organisms which will produce the desired reaction should be used (1). All laboratory prepared media must be tested before use, using organisms expected to grow or not to grow or organisms expected to give a positive reaction or negative reaction. The microorganism selected should be the most fastidious for which the medium was designed. For primary plating media, testing should be done with dilute suspensions of the test organism while undiluted organisms should be used for biochemical media (7). CLSI has certain guidelines for the control organisms used for every medium, the inoculum concentration desired and the growth results expected. Table 1 shows some suggested control organisms, incubation conditions for some bacteriological media with their expected results.

For agar-based media, the qualitative, semiquantitative or quantitative method can be used in testing for growth supporting9. Examples of the qualitative method is the spread plate, for the semiquantitative method econometric and spiral plate while that of the quantitative are pour plate, spread plate and the modified Miles-Misra also known as the drop count technique $(1,10)$. Quantitative assessment is more useful in testing the performance of selective or inhibitory media such as Thayer Martin agar (1).

Quantitative assessment is more difficult to apply in liquid media, hence it is done using endpoint determinations and kinetic parameters. The end point determination focuses on the final result of growth which is seen as an increase in biomass. This can be indirectly determined by measuring the turbidity, changes in electrical properties such as the conductance of the broth, estimating the viable numbers within the broth, production of visible metabolic products such as gas, or reactive compounds such as indole.9,10 Examples of methods which utilize endpoint determinations include copious growth method, end point method and most probable number. Kinetic parameters involves comparison of the growth supporting ability of two batches of broth can be done by measuring the growth curves of identical inocula grown side-byside. The growth rate of the challenge organism is then determined either by viable count or spectrophotometrically as a means of comparing the nutritive properties of the media. Alternatively, comparison can be made using the length of lag phases of the same inoculums on the test and control broths. This method is extremely labour intensive, subject to variability and difficult to implement (10).

\section{Media Storage and Expiry}

Media that are yet to be declared fit for use should be quarantined, tagged unqualified and ideally placed in a storage room separate from that where qualified media is stored. An alternative is tagging and placing them in a clearly identified area within the same room. All QC checks on the quarantined media must be completed before its release for use. Storage conditions of quarantined media should be similar to that of qualified media (10).

Prepared media should be stored away from sunlight and heat. Media containing blood, antibiotics and other organic additives should be stored in the refrigerator. When stored in a cool dry place, the shelf life of prepared media depends on the type of container used. The shelf lives typically are: 2 weeks for media in tubes with loose caps, 3 weeks for media in tubes with cotton wool plugs, 4 weeks for media in Petri dishes (if sealed in plastic bags) and 3 months for media in containers with screw-caps (2).

TABLE 1: PERFORMANCE TESTS ON COMMONLY USED MEDIA

\begin{tabular}{|l|l|l|l|}
\hline Medium & $\begin{array}{l}\text { Control } \\
\text { Organism }\end{array}$ & $\begin{array}{l}\text { Incubation condition and } \\
\text { duration }\end{array}$ & Expected Result \\
\hline Chocolate agar & H. influenza & $\mathrm{CO} 2,24$ hours & Growth \\
\hline Blood agar & S. pyogenes & $\mathrm{CO} 2,24$ hours & $\begin{array}{l}\text { Growth } \\
\text { haemolysis }\end{array}$ \\
\hline & S. pneumonia & $\mathrm{CO}, 24$ hours & $\begin{array}{l}\text { Growth } \\
\text { haemolysis }\end{array}$ \\
\hline $\begin{array}{l}\text { Salmonella-Shigella agar or Deoxycholate } \\
\text { citrate agar }\end{array}$ & E. coli & 24 hours & No growth \\
\hline & S. Typhimurium & 24 hours & Colourless colonies \\
\hline Selenite broth & S. Typhimurium & 24 hours & Growth after subculture \\
\hline & E. coli & 24 hours & $\begin{array}{l}\text { No } \\
\text { subculture }\end{array}$ \\
\hline Bile-aesculin agar & E. faecalis & 24 hours & Growth and blackening \\
\hline & S. pyogenes & 24 hours & No growth \\
\hline
\end{tabular}

Courtesy: WHO, Basic Laboratory Procedure in Clinical Bacteriology. 1991 
Documentation of the performance and sterility of all prepared media must be done and records must be maintained for 2 years (2). For user prepared media, QC forms should contain the quantity prepared, source of the ingredient, lot number, sterilization date, preparation date, expiration date and the name of the person who prepared the media (4).

For commercially prepared, ready-to-use media, sample of the lot should be tested for sterility, usually $5 \%$ of any lot is tested in a batch of 100 units or less while a maximum of 10 units are tested in larger batches. Any one shipment of a product with the same lot number is considered a batch, it is considered a different batch if a separate shipment with the same lot number of a product is received and should be tested separately (4). Following the collection of data over several years by the CLSI Subcommittee on media quality control regarding the incidence of QC failures of commonly used microbiology media, a list of media which did not require retesting if purchased from a manufacturer who follows the CLSI guidelines. However, a written assurance stating that CLSI standards were followed must be supplied by the manufacturer and this verification must be maintained in the laboratory along with its QC protocol. This written assurance must be retained for as long as the laboratory uses the specified media. Some media however need to be retested by the laboratory due to the complexity or failure rate history of the media. Examples include chocolate agar and selective media for pathogenic Neisseria and Campylobacter. Each shipment must also be inspected for cracked media, unequal filling, excessive bubbles, clarity, freezing( seen as a crystalline pattern on the surface of the medium), presence of leakage and visible contamination.4 Blood based media should be checked for signs of haemolysis. There should be no deviation from the normal colour of the media. It should be free of moisture but should have no signs of drying at the edges, Petri dishes should have not be broken or cracked (7). The results of media observations should be recorded along with their lot numbers. When a medium does not meet the standards, corrective action must be taken and this should be documented on a separate record called media failures $\log$.

In antimicrobial susceptibility testing, variability in the content of the media can affect the accuracy of the results obtained. Mueller-Hinton agar will be used in this article as it is best media for routine antimicrobial susceptibility testing of non-fastidious bacteria. Fastidious bacteria such as Haemphilus influenza, Neisseria gonorrhoeae, Neisseria meningitides and
Stroptococcus pneumoniae do not grow well on unsupplemented Mueller-Hinton agar hence they require supplements or different media for their growth. Quality control of a batch or lot of Mueller Hinton is done by testing each batch or lot of agar plates or disks with the appropriate quality control strains to determine if the sizes of the zones of inhibition obtained within the batch fall within the expected range. The acceptable zone diameter quality control limits for a single quality control test (single test/single-organism combination) are listed in CLSI M100-S22, Tables 3A and 3B. For non-fastidious bacteria, significant variations might exist in the results obtained with some batches of Mueller-Hinton agar due to its inability to support adequate growth of the test organism. Factors which can result in such variations include:

1) Cation content: increase in $\mathrm{Ca} 2+$ and $\mathrm{Mg} 2+$ contents cause a false resistance of $P$. aeruginosa to the aminoglycosides and a false resistance of microorganisms to tetracyclines and vice versa. $\mathrm{Ca} 2+$ and/ or $\mathrm{Mg} 2+$ content can be verified by performing antimicrobial susceptibility test on Pseudomonas aeruginosa ATCC 27853 and obtaining correct zone diameters with the aminoglycosides. Variation in the $\mathrm{Ca} 2+$ also affects the results of daptomycin as low calcium content causes a false resistance to daptomycin and vice versa. The recommended $\mathrm{Ca} 2+$ content in Mueller-Hinton agar for optimal daptomycin activity is $50 \mathrm{mg} / \mathrm{L}$ which is similar to the physiological levels. Excess zinc ions in the medium may reduce the sizes of the zones of inhibition of carbapenems (11).

2) $\mathrm{pH}$ : The acceptable $\mathrm{pH}$ range of MuellerHinton agar is 7.2-7.4 at room temperature. The $\mathrm{pH}$ of each batch of the medium should be checked before and after gelling. A reduction in the $\mathrm{pH}$ of the media gives a false resistance of microorganisms to aminoglycosides, clindamycin, macrolides, quinolones and a false susceptibility of microorganisms to the penicillins and tetracyclines and vice versa. The $\mathrm{pH}$ of the media can be checked by macerating enough agar to submerge the tip of a $\mathrm{pH}$ electrode, by allowing a small amount of agar to solidify around the tip of a $\mathrm{pH}$ electrode in a beaker or by the use of a surface electrode. Incubation of the medium in $\mathrm{CO} 2$ should be avoided as it can result in a decrease in the $\mathrm{pH}(11)$.

3) Thymidine and thymine content: The thymidine content in Mueller-Hinton agar 
should be as low as possible. Mueller-Hinton agar with excessive thymidine or thymine content can reverse the inhibitory effect of sulfonamides and trimethoprim thereby causing a false resistance to sulfonamides, trimethoprim, and trimethoprimsulfamethoxazole. Evaluation of the thymidine or thymine content in MuellerHinton agar can be done by testing trimethoprim-sulfamethoxazole disks against Enterococcus faecalis ATCC 29212 or E. faecalis ATCC 33186. Clear, distinct zones of inhibition $\geq 20 \mathrm{~mm}$ is seen in a satisfactory medium while unsatisfactory medium will have no zone of inhibition, growth within the zone of inhibition or a zone of inhibition< $20 \mathrm{~mm}$ (11).

4) Moisture (when using Bauer-Kirby test): The surface of the medium should be moist but droplets of moisture should not be apparent on the surface of the medium or on the Petri dish covers during inoculation of the plates. Plates with excess moisture should be placed in an incubator at $35^{\circ} \mathrm{C}$ or in a laminar flow hood at room temperature with lids ajar until excess moisture is removed by evaporation. This usually takes about 10 to 30 minutes $(11,12)$.

5) Agar depth (when using Bauer-Kirby test): the media should have a uniform depth of about $4 \mathrm{~mm}$. This corresponds to $60-70 \mathrm{ml}$ of medium for $150 \mathrm{~mm}$ diameter plates and 25 to $30 \mathrm{ml}$ for $100 \mathrm{~mm}$ diameter plates $(11,12)$.

\section{QUALITY CONTROL OF VIROLOGICAL MEDIA}

Isolation of viruses is still the gold standard in the diagnosis of infections due to viruses however, not all viruses are culturable. Viral cultures are expensive not only in terms of media, reagents and disposables but also in the time taken to propagate and prepare cell lines, extract the virus from the sample, apply the viral extract to the cell line and diagnose the outcome of the assay. Hence, it is essential to maintain strict quality control in order to maximize the performance of the cell line and limit negative effects of variables which can directly or indirectly affect the assay outcome. There are three methods used in the isolation of viruses in diagnostic virology namely: cell culture, animal inoculation and embryonated eggs. The most commonly used method in clinical virology is cell culture as animal inoculation is extremely costly and cumbersome while embryonated eggs are too expensive and inconvenient for use in the routine diagnostic laboratory though they are used for vaccine production (13). Cell culture is technically the culture of cells in vitro. Tissue or organ culture denotes the growth of tissues or organ is preserved. Clinical virologists usually use these terms interchangeably but cell culture is technically more correct. Previously, a lot of diagnostic virology laboratories initiated and propagated their own cell lines but now there are several commercially prepared cell lines which are more convenient and are commonly purchased. Quality is important in all aspects of cell culture as the quality of materials i.e. cell lines, media and other reagents affects the quality of the cultures and products derived from them. Hence, the main areas of concern in quality control of cell culture are: quality of reagents and material; provenance and integrity of the cell lines and avoidance of microbial contamination. The work/laboratory environment, equipment and personnel also affects the quality of virological media produced hence a brief mention on factors peculiar to the virology laboratory will be mentioned.

\section{Facility Design}

The virology laboratory space must be physically separate from the microbiology laboratory space with dedicated equipments and biosafety cabinets (minimum of level 2). Temperature must be maintained at $22-26^{\circ} \mathrm{C}$ and relative humidity of 30 $50 \%$. There should be dedicated areas for reagent preparation, sample preparation and analysis. There should be dedicated hoods/biosafety cabinets for the preparation of cell lines, sample processing during viral analysis by tissue culture (14). Work surface areas must be made of material allowing regular decontamination. Waste should be segregated properly and disposed by following standard hospital infection control policies (15).

\section{Reagents, Supplies and Equipment}

Supplies of items such as glassware, plastic ware, reagents, commercially prepared culture media and laboratory equipment should be of high quality because they are crucial to the quality of results obtained. There should be consistent supply of all items and technological support of equipment. Quantity, lot number, source and date of receipt should be entered into a log book upon receipt. All supplies and consumables should be stored in appropriate conditions specified by the manufacturer.14 The reagents and materials are potential sources of contamination hence materials of good quality free from microbial contamination and breakage should be used. Materials and reagents for cell culture can be obtained from manufacturers who carry out a range of quality control tests screening for microbial contamination such as Mycoplasma and bovine viral diarrhoea virus (BVDV) (16). 
Sterility testing should be carried out on cell culture flasks prior to their use by using a nonselective, antimicrobial free cell culture media. For prepared media, pyrogen-free water should be used, preferably Type 1 reagent grade water and this must be used as soon as it is produced because of leaching of metals or organic contaminants from the storage container into the water, bacterial contamination as well as a decrease in the resistivity which will occur (16).

Sanitization of $\mathrm{pH}$ probes, hoses, pumps and filter housings should be done using $0.525 \%$ solution of sodium hypochlorite which must be followed by neutralization using $1 \mathrm{M}$ solution of sodium thiosulfate. Fine instruments should be frequently serviced and calibrated by accredited service agencies (16).

Media: Every lot of culture media and supplements must be tested to confirm the support of the growth of the cell line and propagation of viruses are not affected negatively in the various assay formats. Media supplements such as serum or semi-synthethic serum surrogates which can affect the impact of cell growth and virus propagation must be evaluated prior to usage. A new lot of serum should be tested with all cell lines to evaluate the overall growth characteristics. Each lot of serum can be spiked with the various virus stock cultures in order to ensure it is not inhibitory to the virus infection, multiplication and it allows for production of sufficient virus titer. This evaluation should be done well in advance of exhaustion of the current lot of supplements and media. The entire process might take 4-6 weeks and consume laboratory resources hence sufficient time and careful planning is needed (14).

There may be significant variations in the sensitivity to virus isolation and this may depend on the cell subline or clone and the number of times passaged. Hence, information on a particular cell line including the source, type, number of times passaged, confluency and cell condition should be recorded. There should be provisions for back-up of routinely passaged cells in case of contamination or laboratory accident in order to avoid severe disruption of workflow. This back up can be done by freezing and storage of low passage cells at $-70^{\circ} \mathrm{C}$, use of aired stock flasks or carrying of a parallel set of stock flasks using a separate set of tissue culture reagents and glassware.

Most reagents and media used in cell culture can be sterilized either by autoclaving (for heat stable components e.g. water, salt solutions, amino acid hydrolysates) or membrane filtration (for heat labile components). The appropriate type of filter membrane should be selected for use. Cellulose acetate membranes are used for applications involving low protein binding while cellulose nitrate membranes are used for general purpose filtration. The pore size of the membrane should be $0.22 \mu \mathrm{M}$ in order to achieve sterilization. Thick cotton membranes or pads which have larger pore sizes can be used for pre-filtering.16 Sterility testing of the media should be done after filter sterilization. Aliquots of the media are obtained and examined for bacterial and fungal contamination. This should be done daily for 5 days and free from contamination before the lot is declared fit for use. Aliquots of all media components and supplements such as foetal calf serum and L-glutamine should also be checked for sterility (11).

Several organisms such as bacteria, yeasts and fungi may contaminate cell cultures. They are detected as turbidity or a change in the $\mathrm{pH}$ of the culture medium. However, organisms such as Mycoplasma and viruses do not cause turbidity or change in $\mathrm{pH}$. They may or may not cause cytopathological effects (CPE) in the cell line. Thus, they are difficult to detect and may be passaged indefinitely without being detected. Cell lines which are notorious for adventitious virus contamination include primary rhesus monkey kidney cells, primary bovine kidney cells and primary African green monkey kidney cells. Hence cell lines must be tested for microbial contamination prior to use. This can be done by screening the cell lines can be screened for Mycoplasma contamination using DNA specific fluorescent staining and further confirmed by culture. Also available are commercial testing services for Mycoplasma and viruses such as MycoTect test (14).

Cell lines prepared by the laboratory should be subjected to daily growth rate and contamination checks, every month, Hoechst stain should be used to detect Mycoplasma contamination and the sensitivity of the cell line to viral isolation should be monitored by periodic TCID50 experiments with stocks of reference virus.15 Mycoplasma contamination can also result in altered growth rate, morphological changes, chromosome aberrations or alteration in amino acid and nucleic acid metabolism. Daily microscopic examination of the cultures ensures early detection of contamination and initiation of appropriate action. Close observation of the medium for colour changes is important as it indicates an increase or decrease in $\mathrm{pH}$ and rate of metabolism, this is essential as it determine if the cells need feeding or subculturing as epithelial cell lines such as HeLa and Hep2 have a rapid metabolism. Fibroblast cells such as MRC5 and WI-38 have a slower metabolism and therefore need feeding at least once a week and subculturing only once a week (16). Commercially prepared cells should be certified to be free from Mycoplasma, fungal and bacterial contamination and examined for contamination upon receipt. 
Instruction sheets should accompany cells purchased from cell repositories as they contain recommendations on the appropriate medium, subculture procedures, correct seeding concentrations, feeding schedule and safety concerns. There should be daily checks of cells to ascertain growth rate and contamination. During use, cell lines should be handled separately and the cabinet decontaminated in between. Chances of contamination can be further minimized by the use of separate apparel, reagents and glassware for cell culture and exclusion of laboratory with infectious diseases from handling cell culture (14). Contaminated cell culture should be discarded upon detection and the work continued with earlier stocks known to be free of contaminants or obtain fresh stocks from a recognized source.

During cell culture, cells adherent to the cell culture flask can either be detached physically or chemically. In physical detachment, disposable cell scrapers should be used. This is done by removing the growth medium after which the cells are physically detached by scraping the cells from the surface of the flask. Chemical detachment involves the use of enzymes (such as trypsin, pronase or collagenase) and chelating agents (such as ethylene-diamine-tetraacetic acid or versene) for the detachment process. Care must be taken when handling cell monolayers as the enzymes used in the detachment may be toxic to some cells. Some enzymes also remove important receptors and cell surface molecules which are important hence the effects on the enzymes on the chosen cell line must be known prior to its use (13).

\section{Personnel and Environmental Monitoring}

Individuals working with cell culture should wash hands upon entry into the laboratory in order to remove dry skin and loosely adherent microorganisms which are potential sources of contaminants. Surgical gloves and gowns must be worn and gloves must be frequently swabbed with $70 \%$ (v/v) sterile isopropanolol. Cabinets should be stocked with all materials needed in a clutter-free manner prior to the commencement of any procedure. All items that enter the biosafety cabinet must be sprayed with $70 \%(\mathrm{v} / \mathrm{v})$ sterile isopropanolol to prevent dust and particles from entering into the cabinet. Avoid creating bubbles or generating aerosols in the medium or pipette as they can act as sources of contamination. Preferably, a pipette should be used only once and clean spills with $70 \%(\mathrm{v} / \mathrm{v})$ sterile isopropanolol immediately they arise. Personnel should be with proven expertise. They should have training and experience in media and reagents preparation, preparation of dilutions, cell line manipulation, handling harvesting and distribution of cells, maintenance of cell cultures and the outcome of assays. They should be accurate in documentation. Poorly trained staff can also in core areas such as good laboratory practice and aseptic techniques are potential sources of contamination of the media especially during manipulation. They should undergo re-training. The training and experience of staff and laboratory activities documentation enhance quality of results obtained in the virology laboratory (14).

The importance of ensuring the quality of media medical microbiology and virology laboratory cannot be overemphasized as media of low quality will lead to problems with every aspect of the laboratory operation. For accurate and acceptable isolation of pathogens, QC testing of media using a standard protocol helps save time and resources. As the time and resources spent on ensuring production of quality media will be amply repaid in terms of reproducibility of data and reduction on the time spent on investigations of non-conforming results.

\section{INTERNAL QUALITY CONTROL}

The issue of quality control (QC) in the medical microbiology laboratory is complex1. Quality control is defined as all measures put in place to ensure the medical reliability of laboratory data1,2. Quality control is now recognized as being a part of a larger program referred to as 'Quality assurance' (2).

Quality control activities that take place must be recorded to prove their existence1,2. The responsibility for QC usually rest with one person, but in reality, everybody in the laboratory must participate if a program is to be successful1,2. QC programs for the medical microbiology laboratory should include procedures for control of media, temperature, reagents, equipment, susceptibility testing, and all personnel $(1,2)$.

\section{Temperature}

Temperature checks should be done daily on all temperature dependent equipments like water bathes, incubators, refrigerators, heating blocks and freezers 17,18

Thermometers in incubators and refrigerators are easier to read when they are permanently immersed in glycerol $(17,18)$. Glycerol helps prevent temperature fluctuations that occurs when the door is opened to read the thermometer17,18. Each thermometer must be checked against a reference thermometer from the National Institute of Standards and Technology (NIST) before each use $(17,18)$. A large batch of thermometers can be checked at the same time and at the temperature ranges likely to be used $(17,18)$. Thermometers can also be calibrated by 
batch on arrival to the laboratory and once the thermometer has passed calibration it is placed in use17,18. Repeat calibration of in-use thermometers is usually not necessary17,18. Certificates of calibration of the thermometers are kept for the life of the thermometer or until the expiration date on the certificate; after that date, the thermometer can be recalibrated or discarded17,18. For safety reasons, nonmercury thermometers are recommended 17,18 . Mineral spirits with nontoxic, red-dyed alcohol can be used in place of the mercury $(17,18)$.

\section{Equipment Quality Control}

Equipment used in the clinical microbiology laboratory are usually tested for proper performance at intervals as appropriate17,18. The frequency of testing can vary from daily to yearly depending on the equipment in question17,18. A concept termed preventive maintenance is put in place to as an additional control measure17,18. It involves things like replacing filters, oiling and cleaning and instrument recalibration $(17,18)$.

\section{Media Quality Control}

Media prepared in the medical microbiology department must be quality controlled with documentation on their performance and sterility17,18. Records of these documentation must be kept for at least 2 years17,18. Criteria used are generated by the Clinical and Laboratory Standards Institute (CLSI). Conditions such as moisture; to be sure that plates are moisture free before use and also, ensure that there are no signs of drying around the edges of the media17,18. Culture plates should be free of any form of contaminants17,18. Petri dishes should not be cracked or broken17,18. The appearance of media, blood-based plates for example, should not show signs of hemolysis, and also, other plate should not deviate from the normal color and should this happen, it should not be used $(17,18)$.

\section{Reagent Quality Control}

Reagents like oxidase, kovacs, all stains, catalase, $X$ and V strips, Voges-Proskauer and optochin should be checked regularly using negative and positive controls $(17,18)$.

\section{Antimicrobial Susceptibility Quality Control}

Guidelines for the control of susceptibility testing is provided by CLSI, also provided, is a list of control strains from the American Type Culture Collection (ATCC) 17,18. The variables that need to be quality controlled to ensure release of quality reports include instrument failure, antibiotic potency, agar depth, $\mathrm{pH}$, evaporation, inoculum concentration, temperature, moisture, difficulty in determining endpoints $(17,18)$.

\section{Personnel Competency}

Proficiency testing is usually done to ascertain the level of competence of each personnel for each test he or she is expected to perform17,18. Review of work sheet, written examination and direct observation are also ways to determine personnel competence17,18. Tests done on patient samples must pass through proficiency testing at least twice a year17,18. Another form of quality control is the act of reviewing the work of a technologist by another, usually a senior technologist17,18. Encouraging this practice ensures that mistakes are easily caught prior to release of a result17,18. Documentation of test of competency for each employee must be done on employment and yearly from then on and a proof of competency must be kept in his or her personnel file $(17,18)$.

\section{Stock Cultures}

These can be obtained from various sources including commercial sources, patient isolates, ATCC and proficiency testing isolates17,18. Stock cultures are best grown in large volume and divided into small quantities that can be stored for at least a year in order to achieve best results $(17,18)$. This reduces the risk of organisms mutating during repeated subculturing. Stock cultures can also be stored using storage beads. This has the advantage of thawing a single bead and the only down side is its higher cost margin $(17,18)$. All organisms stored in a freezer should be at $-70^{\circ} \mathrm{C} 17,18$. They can also be lyophilized or frozen in liquid nitrogen $(17,18)$.

\section{Quality Control Manual}

This is a document that contains all rules and procedures for QC available in written form at the work stations for all employees $(17,18)$. This document must be signed after review annually and subsequently revised by as supervisor as may be needed $(17,18)$.

\section{CONTROL OF IN-USE ANTISEPTICS}

The importance of laboratory safety in the medical microbiology laboratory cannot be overemphasized19,20. The attendant risk of an individual being exposed to an infectious agent while working in the medical microbiology laboratory increases with the type of the organism, frequency of working on it and level of contact with the infectious agent19,20. This is why there must be appropriate systems in place to ensure antisepsis in th laboratory and also ensure the continuous control of these in-use antiseptics in the medical microbiology laboratory $(19,20)$. 
An antiseptics' effectiveness against the expected spectrum of pathogens is a prerequisite for an antiseptic agent (19-21).. Antiseptics used in the medical microbiology laboratory includes alcohols and iodine $(19,20)$.

Ethyl alcohol and isopropyl alcohol are the two most effective alcohols used19-21. A solution of an alcohol that is to be used for antiseptic purposes is usually filtered through a $0.22-\mu \mathrm{m}$ filter to remove any spores that may be present (19-21). Alcohols are not sporicidal, so, a sterile cotton ball should be used when they are used to saturate cotton balls to be used to prepare the skin for blood collection or inoculation (19-21). Alcohols should only be used in concentrations between $60 \%$ and $90 \%$ and, they must be allowed to evaporate from the surface to which they were applied for them to be effective19-21. The presence of organic materials easily inactivates alcohols (19-21).

Iodine

Iodine tinctures are alcohol and iodine solutions, used mainly as antiseptics and povidone-iodine is the best known iodophor used in the medical microbiology laboratory19,20. Povidone-iodine provides a slow and continuous release of free iodine19,20. They are commonly used as skin preparations from sites where blood is to be drawn19,20. It is important that there is proper amount of contact time when using it as it usually requires more than 30 seconds 19,20.. Iodine tinctures and iodophors must be completely removed from the skin to avoid irritation of the skin (19-21).

\section{Quality Control Programs in the Medical Mycology Laboratory}

The field of medical mycology has rapidly advanced over the last few decades, with tentacles twinning on many specialities of clinical medicine. The spectrum of diseases caused by fungi range from asymptomatic/mild mucocutaneous diseases e.g. dermatophytoses and vulvo-vaginal candidiasis, to chronic debilitating e.g. mycetoma and chronic pulmonary aspergillosis (CPA), to severe lifethreatening invasive fungal disease (IFD) e.g. cryptococcal meningitis. In total, more than 1.5 million people die annually of fungal diseases worldwide22. Aspergillus spp., Cryptococcus spp., Candida spp., and Pneumocystis jirovecii are the main etiologic pathogens of IFD worldwide. Other clinically significant pathogens include the agents of mucormycosis, endemic mycoses, the dermatophytes, and other yeasts and moulds (23).

Timely and accurate diagnosis of IFD is essential but challenging because of the nonspecific clinical and radiographic findings, underlying co-morbid conditions that impedes potentially definitive diagnostic procedures24. Culture and culture-based methods of diagnosis allow species level identification and antifungal susceptibility testing, however, conventional cultures have low yield (24). Culture-independent serum antigen detection tests, such as the $\beta-1,3-D$-glucan (BDG) assay- a "panfungal" biomarker, Aspergillus-specific IgG - a key assay for the serological diagnosis of CPA, cryptococcal antigen (CrAg) tests - a lateral flow assay that has revolutionised the screening and diagnosis of cryptococcal disease, and galactomannan (GM) index-for the diagnosis of invasive pulmonary aspergillosis, may allow earlier diagnosis of IFD than is otherwise feasible with traditional methods (25-27).

Early initiation of antifungals is key to survival and recovery. Therapeutic drug monitoring (TDM) allows treatment optimisation by ensuring therapeutic antifungal serum levels. Available methods for antifungal TDM includes the bioassay, high performance liquid chromatography, liquid chromatography-mass spectrometry and tandem mass spectrometry, each with its own advantages and disadvantages28. Bioassay is the preferred method to measure flucytosine and itraconazole levels. Quality control assessment is a key requirement for triazole TDM (29).

The Global action Fund for Fungal Infections in its action plan aims at developing a more regional or continental EQA schemes for medical mycology, with a focus on assessing immunoassays (antibody and antigen tests), and molecular assays (30), these schemes should also include antifungal drug monitoring. Thus, the aim of an external assurance scheme in medical mycology would be focused at assessing (31,32);

1) Processing of clinical specimens for the diagnosis of fungal diseases

2) The actual identification of the pathogenic fungi (yeast or mould) isolated

3) Interpretation of results of identification (colonisation?, contaminant? or true infection?)

4) Serological and biomarker tests for invasive fungal diseases

5) Antifungal susceptibility testing of pathogenic fungi

6) Methodologies and techniques of therapeutic drug monitoring, and

7) Molecular and proteomic methods of identification of pathogenic fungi. 
The ultimate goal of the exercise is to ensure that the laboratory results that are being reported are accurate, reliable and reproducible33. The internal control programme consists of various tests to check the reagents including the culture media and other consumable materials34. In the laboratory, reference strains have to be provided and, preparation of reagents are needed (34).

Competence of the technical staff in the laboratory can also be maintained through the identification process of unknown fungal strains 34 . The use of the international standards to identify competence in the laboratory results has improved the confidence and acceptance of laboratory results worldwide32. This can also be used in identifying the training needs of members of technical staff as well as evaluating and improving the performance of the laboratory31. However, issues have been raised considering the reliability of these testing procedures which only measure the overt proficiency testing (OPT) targeted at the optimal performance of the laboratory rather than being directed at the routine processes of the patients' samples (35). Another way of improving external quality assurance is to encourage the global registry which usually gives up to date information on rare and emerging fungal diseases (36).

Instructions on every routine procedure being performed must be readily available and it must have been those that have been properly evaluated in a competent manner33. A record of the dates when all media, reagents and stains to be used in medical mycology laboratory was first received, first opened and when to be discarded must be properly recorded (33). Supplies, equipment and the work area in the mycology laboratory must be maintained in an orderly manner to be able to maintain an efficient work flow (33). The equipment used must meet manufactures specifications. In the diagnostic medical mycology laboratory, preservation of the strains of fungi isolated should be kept alive for further evaluation for molecular identification, antifungal susceptibility testing and, epidemiological purposes34. The techniques for this, usually require serial sub-culturing as well as storage in liquid nitrogen, glycerol $(10 \%)$ (at $-20 \circ \mathrm{C}$ and $-80 \mathrm{oC}$ ) and lyophilisation. This also serves as the stock culture for the quality control organisms (31).

A new strain of fungus isolated in the diagnostic medical laboratory should be sent to the reference medical laboratory for further identification and final confirmation. A properly-labelled screw cap tubes with masking tape should be used35. There are different types of fungal culture media that are used for the isolation, identification and maintenance of the fungal strains. The performance of both laboratory and commercially prepared media must be monitored. A record containing the identification of the media, preparation, packaging evaluation as well as sterility check. The medium should be inoculated with the appropriate test organisms that produce negative and positive reactions (33).

On the African continent, a typical example of an EQA scheme is that of the National Health Laboratory Service (NHLS) Proficiency Testing Scheme (PTS) for Mycology, which operates across all NHLS laboratories in South Africa and laboratories in over 20 countries across Africa. NHLSPTS conducts three surveys per year, with each survey including, 4 mould and 1 yeast isolates for identification, slide preparation for microscopy, a simulated blood or cerebrospinal fluid samples for cryptococcal antigen testing, and 1 yeast isolate for identification and antifungal susceptibility testing (37). However, a real world experience on EQAS from seven countries on laboratory practices for diagnosis of fungal infection reported by the Asia Fungal Working Group initiative showed that only $56 \%$ of the labs participated in an EQAS, and only $43 \%$ conducted regular formal staff training (38). There is clearly a need to facilitate improved participation, as this will lead to improved laboratory performance in the mid-term.

Fungal diseases are an emerging threat to the public health worldwide, they are difficult to diagnose and treat. The true burden of fungal diseases in many countries across the world remains unknown; due both non-existence of mycologists and very limited availability of good, dedicated diagnostic mycology laboratories. EQA schemes allow specialist tests to be done outside of the reference laboratory in general laboratories reliably. Furthermore, EQA schemes enhances patient care and safety through improved laboratory practice.

There is need for programs to ensure the comparability of data among testing laboratories across different continents. In the past the differences in quality programmes operated in laboratories made this difficult however Laboratory accreditation programs on quality standards such as EN 45000 series and ISO Guide 25 has facilitated reproducibility in the data produced by different laboratories across the world thus ensuring wider acceptability and reproducibility of the data. The ISO 15189 for Medical Laboratories states that the laboratory shall participate in organised inter-laboratory comparisons, such as external quality assessment schemes, that encompass the extent and complexity of examination procedures used by the laboratory and the laboratory management shall monitor the results of external quality assessment and participate in the 
implementation of corrective actions when control criteria are not fulfilled.

The objectives of an EQA are; to provide a measure for individual laboratory quality, to supplement internal quality control procedures, to provide a measure of the "state of the art" for a test, to obtain consensus values when true values are unknown, to investigate factors in performance (methods, staff etc) and to act as an educational stimulus to improvement in performance. In the case of microbiology EQA, the responses are evaluated against a predetermined intended response (e.g. the identity of the organism and its antimicrobial susceptibility pattern). The key steps towards a successful EQA as outlined by the Centre for Disease control involves the following steps occurring in a continuous cycle; defining the

\section{REFERENCES}

1 Kumari S, Bhatia R. Quality Assurance in Bacteriology and Immunology. 2003

2 World Health Organization. Basic Laboratory Procedures in Clinical Bacteriology. 1991.

3 Basu S, Pal A, Desai PK. Quality Control of Culture Media in a Microbiology Laboratory. Indian J Med Microbiol 2005; 23: 159-163.

4 Tille PM. Bailey and Scott's Diagnostic Microbiology. 13th ed. Elsevier: Missouri, 2014.

5 USP. Microbiological Best Laboratory Practices. Pharmacopeial Forum 2004; 30: 1713-1721.

6 Basu S, Pal A, Desai PK. Quality control of culture media in a microbiology laboratory. Indian J Med Microbiol 2005; 23: 159-163.

7 Misra S. Performance Improvement in the Microbiology Laboratory. In: Textbook of Diagnostic Microbiology. Elsevier: Missouri, 2015, pp 93-109.

8 Duguid JP, Collee JG, Fraser AG, Aikman KW. Organization of the Clinical Bacteriology Laboratory; Quality Assurance. In: Mackie and McCartney Practical Medical Microbiology. Churchhill Livingstone: London, 1996, pp 1-16.

9 Weenk GH. Microbiolological assessment of culture media: comprarison and statistical evaluation of methods. In: Cuture mMedia for Food Microbiology. Elsevier: Missouri, 1995, pp 1-22.

10 Sutton S. Quality Control of Microbiological Culture Media. 2006.

11 CLSI. M100-S27 Performance Standards for Antimicrobial Susceptibility Testing. 2017.

12 CLSI. M02-A11. Performance Testing for Antimicrobila Disk Susceptibility Tests; Approved StandardEleventh Edition. 2012. problems, planning for an EQA, implementing the EQA, follow up the EQA and finally to review and strategically plan the EQA.

\section{EXTERNAL QUALITY CONTROL}

With less restriction on trade throughout the world the comparability of data among testing laboratories becomes all the more important. In the past the differences in quality programmes operated in laboratories made this difficult. Laboratory accreditation to quality standards such as EN 45000 series and ISO Guide 25 [4] has given creditability to data produced by laboratories and has ensured wider acceptability of the data. Many European countries with national accreditation bodies now have multilateral recognition agreements

13 Jerome KR. Lennette's Laboratory Diagnosis of Viral Infections. 4th ed. CRC Press: New York, 10.

14 Danielson RE. Quality Control for Virological Analysis. In: Manual of Environmental Microbiology. ASM Press: Washington, 2016, p ch2.5.4.

15 Tibbets MW, Gomez R, Kannangai R, Sridharan G. Total Quality Management in Clinical Virology Laboratories. Indian J Med Microbiol 2006; 24: 258-262.

16 George VG, Hierholzer JC, Ades EW. Cell Culture. In: Virology Methods Manual. Academic Press: London, 1996, pp 3-24.

17. Mahon CR, Lehman DM, Manuselis G. Textbook of Diagnostic Microbiology. 5th ed. Missouri: Elsevier Saunders; 2015.94-104

18. Procop GW, Church DL, Hall GS, Janda WM, Koneman EW, Paul CS, et al. Koneman's color atlas and textbook of diagnostic microbiology. 7th ed. Philadelphia: Wolters kluwer health; 2017. 46-65.

19. Mahon CR, Lehman DM, Manuselis G. Textbook of Diagnostic Microbiology. 5th ed. Missouri: Elsevier Saunders; 2015.60-71.

20. Bennett JE, Dollin R, Blasser MJ. Mendell, Douglas, and Bennett's Principles and practice of infectious diseases. 8th ed. Philadelphia: Elsevier saunders; 2015.32943309.

21. Boyce JM, Dupont HL, Massaro J, Sack D, Schaffner DW. An expert panel report of a proposed scientific model demonstrating the effectiveness of antibacterial handwash products. Am J Infect Control;2012,40(8):742-749.

22. Bongomin F, Gago S, Oladele R, Denning D. Global and Multi-National Prevalence of Fungal DiseasesEstimate Precision. J Fungi [Internet]. 2017 Oct 18;3(4):57. Available from: http:/ / www.mdpi.com/2309-608X/3/4/57

23. Chastain DB, Henao-Martínez AF, Franco-Paredes C. Opportunistic Invasive Mycoses in AIDS: Cryptococcosis, 
Histoplasmosis, Coccidiodomycosis, and Talaromycosis. Curr Infect Dis Rep [Internet]. 2017 Aug;19(10):36. Available from: https://doi.org/10.1007/s11908-017-0592-7

24. Clancy CJ, Nguyen MH. Finding the missing 50\% of invasive candidiasis: How nonculture diagnostics will improve understanding of disease spectrum and transform patient care. Clin Infect Dis. 2013;56(9):1284-92.

25. Hoenigl M, Prattes J, Spiess B, Wagner J, Prueller F, Raggam RB, et al. Performance of galactomannan, beta-dglucan, aspergillus lateral-flow device, conventional culture, and pcr tests with bronchoalveolar lavage fluid for diagnosis of invasive pulmonary aspergillosis. J Clin Microbiol. 2014;52(6):2039-45.

TDM) of antifungal agents: Guidelines from the british society for medical mycology. J Antimicrob Chemother. 2014;69(5):1162-76.

29. Bruggemann RJM, Touw DJ, Aarnoutse RE, Verweij PE, Burger DM. International Interlaboratory Proficiency Testing Program for Measurement of Azole Antifungal Plasma Concentrations. Antimicrob Agents Chemother [Internet]. 2009 Jan 1;53(1):303-5. Available from: http://aac.asm.org/cgi/doi/10.1128/AAC.00901-08

30. Denning DW. The ambitious "95-95 by 2025" roadmap for the diagnosis and management of fungal diseases. Thorax [Internet]. 2015;70(7):613-4. Available from: http:/ / thorax.bmj.com/content/early/2015/05/29/thoraxj nl-2015-207305.extract

31. Chander J. Textbook of Medical Mycology. Jaypee Brothers,Medical Publishers Pvt. Limited; 2017.

32. Guzel O, Guner EI. ISO 15189 accreditation: Requirements for quality and competence of medical 1 aboratories, experience of a laboratory I. Clin Biochem. 2009 Mar;42(4-5):274-8.

33. McGinnis MR. Laboratory Handbook of Medical Mycology. Elsevier Science; 2012.
26. Lahmer T, Held J, Rasch S, Schnappauf C, Beitz A, Schmid RM, et al. Usage of 1,3- $\beta$-d-Glucan for Early Detection of Invasive Mycoses and Outcome Parameter in Immunocompromised Critically Ill Patients. Mycopathologia [Internet]. 2016;181(11-12):815-21. Available from: http:/ /link.springer.com/10.1007/s11046-016-0061-0

27. Moura S, Cerqueira L, Almeida A. Invasive pulmonary aspergillosis: current diagnostic methodologies and a new molecular approach. Eur J Clin Microbiol Infect Dis [Internet]. 2018 May 13; Available from: http:/ /link.springer.com/10.1007/s10096-018-3251-5

28. Ashbee HR, Barnes RA, Johnson EM, Richardson MD, Gorton R, Hope WW. Therapeutic drug monitoring (

34. Kauffmann-Lacroix C, Cassaing S, Bessieres M-H, Mayet D, Linas M-D, Roques C. [Quality controls in medical mycology]. J Mycol Med. 2011 Mar;21(1):15-8.

35. Reilly AA, Salkin IF, McGinnis MR, Gromadzki S, Pasarell L, Kemna M, et al. Evaluation of mycology laboratory proficiency testing. J Clin Microbiol. 1999 Jul;37(7):2297-305.

36. Seidel D, Durán Graeff LA, Vehreschild MJGT, Wisplinghoff $\mathrm{H}$, Ziegler $\mathrm{M}$, Vehreschild J, et al. FungiScopeTM -Global Emerging Fungal Infection Registry. Mycoses. 2017 Aug;60(8):508-16.

37. The National Health Laboratory Service (NHLS) Proficiency Testing [Internet]. [cited 2018 Jul 31]. Available from:

http:/ / www.nhls.ac.za/?page=proficiency_testing\&id $=42$

38. Chindamporn A, Chakrabarti A, Li R, Sun P-L, Tan B-H, Chua M, et al. Survey of laboratory practices for diagnosis of fungal infection in seven Asian countries: An Asia Fungal Working Group (AFWG) initiative. Med Mycol [Internet]. 2018 Jun 1;56(4):416-25. Available from: https://academic.oup.com/mmy/article/56/4/416/420167 1 